\title{
Special Section Guest Editorial: The Origins Space Telescope
}

\author{
George Helou a and Antonios Seas ${ }^{\mathrm{b}}$ \\ ${ }^{a}$ Caltech/IPAC, Pasadena, California, United States \\ ${ }^{b}$ NASA Goddard Space Flight Center, Greenbelt, Maryland, United States
}

It is not often that a team of scientists and engineers have the opportunity to start with a blank sheet and design the space observatory of their dreams, subject only to a cost cap, albeit a generous one. Fortunately, they do have it once a decade when the astrophysics community prepares for the Decadal Survey of Astronomy and Astrophysics conducted by the U.S. National Academies. For this decade's round, the dreaming benefited from a more deliberate approach funded by NASA, allowing for flagship space mission design in four broadly defined areas: an $\mathrm{x}$-ray observatory named Lynx to study the very hot and energetic Universe; two ultraviolet to near-infrared observatories, Habex and LUVOIR, emphasizing exoplanets among many other topics; and the subject of this special section, the Origins Space Telescope (Origins), aimed at elucidating myriad questions about the emergence of today's Universe.

The scientific vision of Origins encompasses several key processes mediating the evolution from the early diffuse Universe to the rich and highly contrasted Universe that has come to host human life. The Science and Technology Definition Team for Origins looked to the remarkable results from past missions, especially Spitzer and Herschel, and to expectations from Webb and Roman, as well as ground-based observatories, and evaluated the most promising directions for a future space mission covering the infrared-to-submillimeter spectrum. They converged on a sixmeter class telescope actively cooled to near $4 \mathrm{~K}$ with imaging and spectroscopic instruments enabling a thousand-fold improvement in sensitivity over past missions (Leisawitz et al.), while fitting within the mission guidelines and cost cap. This design is capable of addressing outstanding questions about the formation of first galaxies and the rise of metals and dust in the early Universe, about the development of habitable worlds and emergence of life, and about many topics in between, such as the life cycle of black holes in galaxy centers and their far-reaching effects on their surroundings, the star formation history in small and large galaxies and its mechanics at all scales within galaxies, and the likelihood of life-friendly planets orbiting the most numerous stars in our galaxy.

Evolving such a powerful mission concept within the cost cap and guidelines was a daunting challenge. The trades and decisions leading from the blank screen to the Origins mission concept are recounted by Leisawitz et al., and the threads connecting science drivers to design choices and instrumental capabilities are detailed by Meixner et al. The design adopted for the all-important cryo-thermal system is described in more detail by DiPirro et al. and that of the optical system by Corsetti et al. Also, Petro sets out a notional plan for integration and testing of such an observatory. An adaptation of the James Webb Space Telescope design is offered by Arenberg et al. as an alternative approach to meeting the Origins goals and objectives, including the required thermal performance.

The Origins baseline mission concept features three focal plane instruments. The Origins Survey Spectrometer (OSS) would provide spectroscopy at wavelengths from 25 to $590 \mu \mathrm{m}$ with three modes, ranging from a spectral resolution $\lambda / \Delta \lambda=300$ in a many-arcminute slit of spatial coverage to $\lambda / \Delta \lambda$ exceeding $10^{5}$ a single pixel of a few arcseconds (Bradford et al.). The Far-Infrared Imager Polarimeter (FIP) would offer imaging in pointed or mapping mode and polarimetry at $50 \mu \mathrm{m}$ in a field of view of a few arcminutes, or $250 \mu \mathrm{m}$ in a field of view of a dozen arcminutes (Staguhn et al.). The Mid-Infrared Spectrometer and Camera transit spectrometer (MISC-T) would obtain spectra with resolution $\lambda / \Delta \lambda$ on the order of a hundred covering 2.8 to $20 \mu \mathrm{m}$ in a single pointing, with stability and precision reaching better than $5 \mathrm{ppm}$, enabling the detection of biosignatures in exoplanet atmospheres (Sakon et al.). The Heterodyne

(C) 2021 Society of Photo-Optical Instrumentation Engineers (SPIE) 
Receiver for Origins (HERO) is offered by Wiedner et al. as a potential enhancement to the observatory, providing nine-pixel mapping of selectable narrow spectral bands between 110 and $620 \mu \mathrm{m}$, up to very high spectral resolution of roughly $10^{7}$.

The Origins design relies on a broad array of technologies, most of which were available in 2019 at Technology Readiness Levels (TRL) or 5 or higher. As befits a visionary concept, the design also identifies a small number of technologies in detectors, ancillary detection system components and cryocoolers that need advancing to TRL 5. This special section includes eight papers concerned with Origins technologies at various levels of readiness, addressing either their adaptation or their promise and paths to implementation. DiPirro et al. describe cryocooling technologies. Hailey-Dunsheath et al. discuss Kinetic Inductance Detectors; Nagler et al. discuss Transition-Edge Sensor detectors; and Echternach et al. discuss lowfrequency readout quantum capacitance detectors in large array format. Wollman et al. report on recent advances in superconducting nanowire single-photon detector technology. Roellig et al. describe mid-infrared detector development. Bradley et al. write about the advancement of digital signal processing hardware and algorithms. Tangential to technology but also important, Sandin et al. discuss materials evaluation for Origins.

Astrophysics thrives by looking across the whole electromagnetic spectrum, not to mention other celestial messengers from gravitational waves to cosmic rays, to inform our physical understanding of cosmic phenomena. As a result, NASA and the astronomy community are constantly striving to balance assets and capabilities across the spectrum. The spectral range anchored by Origins is replete with unique information to illuminate many diverse topics, some of which are mentioned above. It offers diagnostics of atoms, molecules, ions, and dust in greater abundance than other spectral ranges; for example, Origins reaches more than a hundred transitions of water vapor (Figure 5 of Meixner et al.), making it uniquely capable of tracing water's progression through various environments and temperatures to an Earth-like planet. Many of the diagnostics remain accessible to Origins over a large fraction of the age of the Universe in spite of redshift, and they are little affected by dust extinction. The design of Origins and its instruments captures the promise of the infrared as demonstrated by its ability to acquire data meeting a diverse and exciting set of science requirements, in the tradition of the NASA Great Observatories. It offers three orders of magnitude improvement in sensitivity over past missions, and the flexibility to respond to community-driven science. This giant leap opens up a potential for discovery much richer than the designers could have anticipated. 